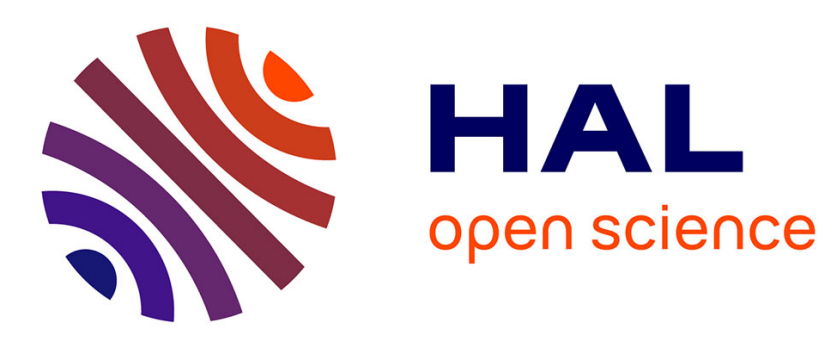

\title{
Low-Sound-Level Auditory Processing in Noise-Exposed Adults
}

\author{
Emanuele Perugia, Christopher Plack, Michael Stone
}

\section{To cite this version:}

Emanuele Perugia, Christopher Plack, Michael Stone. Low-Sound-Level Auditory Processing in NoiseExposed Adults. Forum Acusticum, Dec 2020, Lyon, France. pp.631-632, 10.48465/fa.2020.0481 . hal-03234222

\section{HAL Id: hal-03234222 \\ https://hal.science/hal-03234222}

Submitted on 26 May 2021

HAL is a multi-disciplinary open access archive for the deposit and dissemination of scientific research documents, whether they are published or not. The documents may come from teaching and research institutions in France or abroad, or from public or private research centers.
L'archive ouverte pluridisciplinaire HAL, est destinée au dépôt et à la diffusion de documents scientifiques de niveau recherche, publiés ou non, émanant des établissements d'enseignement et de recherche français ou étrangers, des laboratoires publics ou privés. 


\title{
LOW-SOUND-LEVEL AUDITORY PROCESSING IN NOISE-EXPOSED ADULTS
}

\author{
Emanuele Perugia ${ }^{1}$ \\ Christopher J. Plack ${ }^{1,2}$ \\ Michael A. Stone ${ }^{1}$ \\ ${ }^{1}$ Manchester Centre for Audiology and Deafness, University of Manchester, UK \\ ${ }^{2}$ Department of Psychology, Lancaster University, UK \\ emanuele.perugia@manchester.ac.uk
}

\section{INTRODUCTION}

Few studies have explored the relationship between noise exposure and auditory processing at low sensation levels (SLs). There is evidence that young normal-hearing subjects frequently exposed to high-noise events have poorer envelope discrimination [1], and poorer amplitude modulation detection (AMD) [2], at low SLs compared to low noise-exposed control subjects. Conversely, in another study, although young normal-hearing subjects with extensive use of personal music players had worse frequency discrimination thresholds at low SLs, they had better AMD thresholds compared to a control group [3]. Furthermore, older subjects showed better AMD than young subjects [2].

\section{METHODS}

\subsection{Participants}

Forty participants (20 females) between the ages of 33 and 75 years (mean 58.7 years) were recruited, with normal or near-normal absolute thresholds up to $1 \mathrm{kHz}$ and a mildto-moderate threshold elevation between 3 and $6 \mathrm{kHz}$. The study was approved by the NRES Committee North West - Greater Manchester South (IRAS number 184199; REC number 16/NW/0260).

\subsection{Pure tone audiometry}

Pure-tone air-conduction audiometric thresholds were measured at 11 frequencies between $125 \mathrm{~Hz}$ and $8000 \mathrm{~Hz}$. The average of the hearing thresholds was used to classify participants as Normal (17 participants) with an average of $\leq 20 \mathrm{~dB}$ HL, or Mild Hearing Loss (23 participants) with an average between 21 and $36 \mathrm{~dB}$ HL.

\subsection{Noise exposure}

Lifetime noise exposure was estimated through the Noise Exposure Structured Interview (NESI, [4]), recording the frequency, duration and level of each activity to derive a single measure related to lifetime-accumulated energy of exposure. The NESI score was used to categorise participants into Low-, Medium- or High-Noise groups (40$20-40 \%$ of distribution, respectively). Only the Low- and High-Noise groups were considered in the mixed-effects modelling. These had median NESI scores of 1.05 and $2.23 \log 10$ (Energy), respectively.

\subsection{Musical experience}

The NESI was also used as a proxy measure of participants' musical experience. The total number of hours of playing a musical instrument and/or singing was taken as the metric of musical experience. Twenty-one participants had no musical experience, and 19 had some experience or were expert musicians.

\subsection{Psychophysical tasks}

All testing was performed in a double-walled soundattenuating booth using monaural stimuli delivered by Etymoric Research ER4s. Computer-tracked psychophysical measures of absolute threshold (ABS) were followed by measures of frequency difference limens (FDLs) and AM discrimination (MDD). The experiments were performed at five centre frequencies $(0.75,1,3,4$ and $6 \mathrm{kHz})$ and at both 15 and $25 \mathrm{~dB}$ SL relative to the ABS.

\subsubsection{Absolute threshold}

The ABS was measured for pure-tone signals using a threealternative forced-choice method, with a two-down one-up adaptive tracking procedure.

\subsubsection{Frequency difference limens}

A two-alternative forced-choice paradigm, with a twodown one-up adaptive tracking procedure, was used. One interval contained four identical tone bursts (AAAA), while the other interval contained two alternated (target) bursts (A'B'A'B') with $\Delta f$ increase and decrease in $\mathrm{f} 0$.

\subsubsection{Amplitude modulation depth discrimination}

A two-alternative forced-choice paradigm, with a twodown one-up adaptive tracking procedure, was used. One interval contained the standard (target) AM tone with a modulation depth ( $m s$ ) fixed at $9.6 \mathrm{~dB}$ (peak-valley amplitude), while the other interval contained the comparison AM tone with a modulation depth $(m c)$ initially set at $1.7 \mathrm{~dB}$ (i.e. a very shallow modulation) and was always less than the standard depth. The task was to indicate the interval having the greater modulation depth. The MDD threshold was expressed as $\Delta m=m s-m c$. 


\subsection{Data analysis}

Mixed-effects modelling was performed including only the Low- and High-Noise exposure groups. The entire cohort was used in the Spearman's correlation coefficient for ranked data.

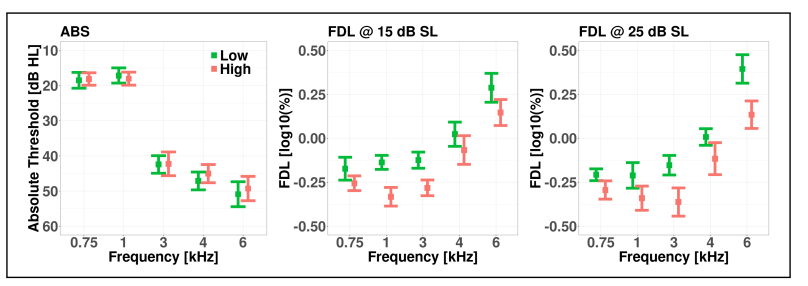

Figure 1. Mean (and SE) of ABS and FDLs for the Lowand High-Noise exposure groups.

\section{RESULTS}

\subsection{Absolute thresholds}

The ABS results (Fig. 1, left panel) were best explained by a mixed model $(\mathrm{R} 2=0.80)$ with Frequency and Hearing groups as fixed effects; while the participants were entered as random intercepts. The thresholds at 0.75 and $1 \mathrm{kHz}$ were significantly lower than at 3,4 and $6 \mathrm{kHz}$, and the 3 $\mathrm{kHz}$ threshold was lower than that at $6 \mathrm{kHz}$. The Normal group had an average threshold about $10 \mathrm{~dB}$ better than the Mild Hearing Loss group.

\subsection{Frequency difference limens}

The FDLs (Fig. 1) were fitted with a mixed model (R2=0.76) having Frequency and Music Experience groups as fixed effects; and including by-participant random intercepts and by-participant random slopes for Frequency. The FDL was significantly worse at 4 and $6 \mathrm{kHz}$ than the lower frequencies. No effect of lifetime noise exposure was observed. Participants without music experience had worse performance than the participants with music experience.

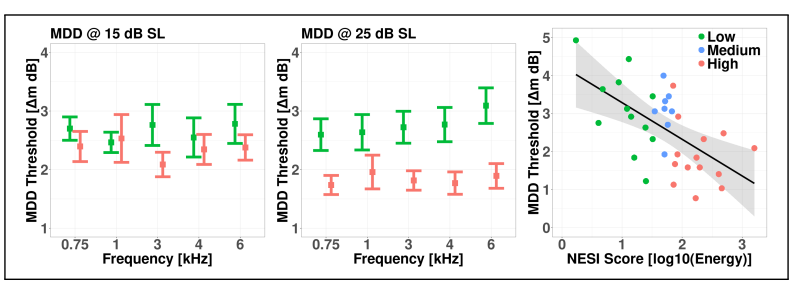

Figure 2. Mean (and SE) of MDD thresholds for the Low and High noise exposure groups. In the right panel: MDD at $6 \mathrm{kHz}$ and $25 \mathrm{SL} \mathrm{dB}$ against the noise exposure scores.

\subsection{Amplitude modulation depth discrimination}

Figure 2 shows the results for the MDD thresholds. The mixed-model (R2=0.47) had SL, Noise groups and their interaction as fixed effects, and Music groups separately as fixed effects; participants were treated as random effects.
Overall performance was lower (better) at $25 \mathrm{~dB}$ SL compared to $15 \mathrm{~dB}$ SL. MDD threshold was better for participants with music experience than for those without. At dB 25 SL, the High-Noise group showed significantly lower MDD thresholds than the Low-Noise group, and a negative correlation existed between NESI score and MDD threshold at $0.75,3,4,6 \mathrm{kHz}$. The latter was the most robust ( $\mathrm{r}=-0.591 p<0.001)$.

\section{DISCUSSION \& CONCLUSIONS}

Two psychophysical tasks were used to assess a possible low-SL sub-clinical hearing deficits as a function of noise exposure. There was a significant effect of participants' musical experience on FDL performances. The FDL increased (worsened) as a function of frequency irrespective of Noise or Hearing Groups. These results suggest that the FDL is not a sensitive marker for noise damage assessment. The MDD threshold at $25 \mathrm{~dB}$ SL was lower (better) for the High-Noise group than for the Low-Noise group. The results support earlier evidence $[3,5]$ of a negative relation between MDD thresholds and noise exposure. Improved performance on MDD is most likely associated with $\mathrm{OHC}$ dysfunction.

\section{REFERENCES}

[1] M. A. Stone, B. C. J. Moore, and H. Greenish, "Discrimination of envelope statistics reveals evidence of sub-clinical hearing damage in a noise-exposed population with 'normal' hearing thresholds," International Journal of Audiology, vol. 47, pp. 737-750, Dec. 2008.

[2] M. A. Stone and B. C. J. Moore, "Amplitudemodulation detection by recreational-noise-exposed humans with near-normal hearing thresholds and its medium-term progression," Hearing Research, vol. 317, pp. 50-62, Nov. 2014.

[3] S. N. Vinay and B. C. J. Moore, "Effects of the use of personal music players on amplitude modulation detection and frequency discrimination," The Journal of the Acoustical Society of America, vol. 128, pp. 36343641, Dec. 2010.

[4] H. Guest, R. S. Dewey, C. J. Plack, S. Couth, G. Prendergast, W. Bakay, and D. A. Hall, "The Noise Exposure Structured Interview (NESI): An Instrument for the Comprehensive Estimation of Lifetime Noise Exposure," Trends in Hearing, vol. 22, p. 2331216518803213, 2018 Jan-Dec.

[5] G. Prendergast, R. E. Millman, H. Guest, K. J. Munro, K. Kluk, R. S. Dewey, D. A. Hall, M. G. Heinz, and C. J. Plack, "Effects of noise exposure on young adults with normal audiograms II: Behavioral measures," Hearing Research, vol. 356, pp. 74-86, Dec. 2017.

This study was supported by the NIHR Manchester Biomedical Research Centre and by the Medical Research Council, UK (MR/L003589/1 and MR/M023486/1). 